\section{Augmentation paradoxale du nombre de synapses glutamatergiques lors des troubles cognitifs légers de la maladie d'Alzheimer}

Karen F.S. Bell, A. Claudio Cuello
Département de Pharmacologie,

Université McGill, 3655,

Promenade Sir William Osler, Montréal, Québec H3G 1Y6 Canada.

claudio.cuello@mcgill.ca
> La maladie d'Alzheimer est la principale cause de démence chez les personnes âgées: elle affecte approximativement 450000 Canadiens âgés de plus de 65 ans. Ses répercussions économiques néfastes sont considérables sur nos sociétés modernes: elles tiennent autant aux coûts du traitement qu'au nombre d'heures de travail perdues par les accompagnants. Au Canada, on estime à environ 5,5 milliards de dollars les sommes dépensées pour traiter les personnes atteintes de la maladie d'Alzheimer et d'affections connexes (données disponibles sur le site http://www.alzheimer.ca/french/ disease/stats-costs.htm). La présence de plaques amyloïdes et de neurofibrilles dans le cortex et l'hippocampe est souvent interprétée comme une manifestation claire de la progression de la maladie. Cependant, contrairement à l'opinion courante, la diminution de la densité des terminaisons synaptiques serait un marqueur plus fiable de la progression de la maladie. En effet, la chute de la densité des terminaisons synaptiques est très étroitement associée à la réduction des capacités cognitives des patients atteints par la maladie d'Alzheimer [1, 2]. Deux altérations structurales des synapses sont néfastes pour la neurotransmission: d'une part, la réduction du nombre de synapses et, d'autre part, l'apparition de neurites dystrophiques (une croissance de l'arborisation ner- veuse qui se reforme anormalement et fonctionne moins efficacement $[3,4]$ ) $[1,2,5]$. C'est pourquoi, afin d'améliorer notre compréhension de la fonction des synapses dans le contexte de la pathologie de l'Alzheimer, notre laboratoire a quantifié la densité de différentes populations de boutons présynaptiques corticaux contenant des neurotransmetteurs spécifiques, ainsi que le taux de neurites dystrophiques dans le néocortex de souris transgéniques utilisées comme modèles de la maladie d'Alzheimer [7]. Ces modèles transgéniques surexpriment le précurseur de la protéine amyloïdique et, par conséquent, ils reproduisent la pathologie amyloïde typique de la maladie d'Alzheimer.

Nous avons récemment mis en évidence que chaque système de neurotransmetteurs montre une élévation de la densité de boutons présynaptiques durant les stades précoces de la maladie, cette élévation est suivie par une déplétion qui s'accentue à mesure que la pathologie progresse $[6,7]$. Nos résultats révèlent qu'il y a une corrélation entre l'évolution de la pathologie amyloïde chez les souris transgéniques et le rôle de divers types synaptiques, correspondant à l'ordre suivant de vulnérabilité : cholinergique > glutamatergique $>$ GABAergique $[6,7]$. Le système cholinergique présente un schéma de remodelage synaptique avant même que ne soit détectable l'entrée en action des systèmes glutamatergique et GABAergique $[6,7]$; soit, respectivement, les systèmes principaux de la transmission synaptique excitatrice et inhibitrice. Le système cholinergique montre aussi une abondance anormale de neurites cholinergiques dystrophiques localisées principalement dans le neuropile interne de la péri-plaque: cette observation accrédite donc l'idée d'une vulnérabilité «première » du système cholinergique à la pathologie amyloïde $[6,7]$ (Figure 1). À la différence du système glutamatergique, le taux de neurites GABAergiques dystrophiques était moindre et n'était affecté ni par la taille des plaques, ni par le stade d'évolution de la maladie dans ces modèles transgéniques $[6,7]$.

Afin d'étendre à la fois la validité de ce schéma de remodelage cortical observé grâce aux modèles transgéniques, et afin de clarifier le rôle du système glutamatergique dans la maladie d'Alzheimer, nous avons approfondi cette recherche sur du tissu humain. En collaboration avec le Religious Orders Study de I'Université Rush (Chicago, États-Unis), nous avons quantifié la densité de boutons présynaptiques glutamatergiques et le taux de neurites dystrophiques dans le gyrus frontal médian de trois groupes de sujets: un groupe formé de personnes ne présentant aucun trouble cognitif, un groupe de personnes avec des troubles cognitifs légers et un groupe de personnes affectées de la maladie d'Alzhei- 
mer à un stade moyen ou avancé. Nos résultats, qui ont été publiés récemment dans le Journal of Neuroscience [8], montrent de façon saisissante que la maladie d'Alzheimer révèle un schéma de remodelage synaptique identique à ceux décrits dans les modèles transgéniques, soit une augmentation du nombre de boutons présynaptiques glutamatergiques durant les troubles cognitifs légers suivie par une forte baisse au fil de la progression de la pathologie (Figure 2). De plus, des corrélations significatives ont été trouvées entre les résultats des tests cognitifs et les densités de boutons présynaptiques glutamatergiques, confirmant le rapport de cause à effet entre la chute de la densité synaptique et la croissance des troubles cognitifs. Notre étude a également mis en évidence la présence de nouvelles neurites dystrophiques glutamatergiques dont l'incidence va de pair avec la taille des plaques (Figure 2). Nos observations montrent une période d'élévation de terminaisons synaptiques durant les stades précoces de la maladie d'Alzheimer; ce constat concorde avec les résultats

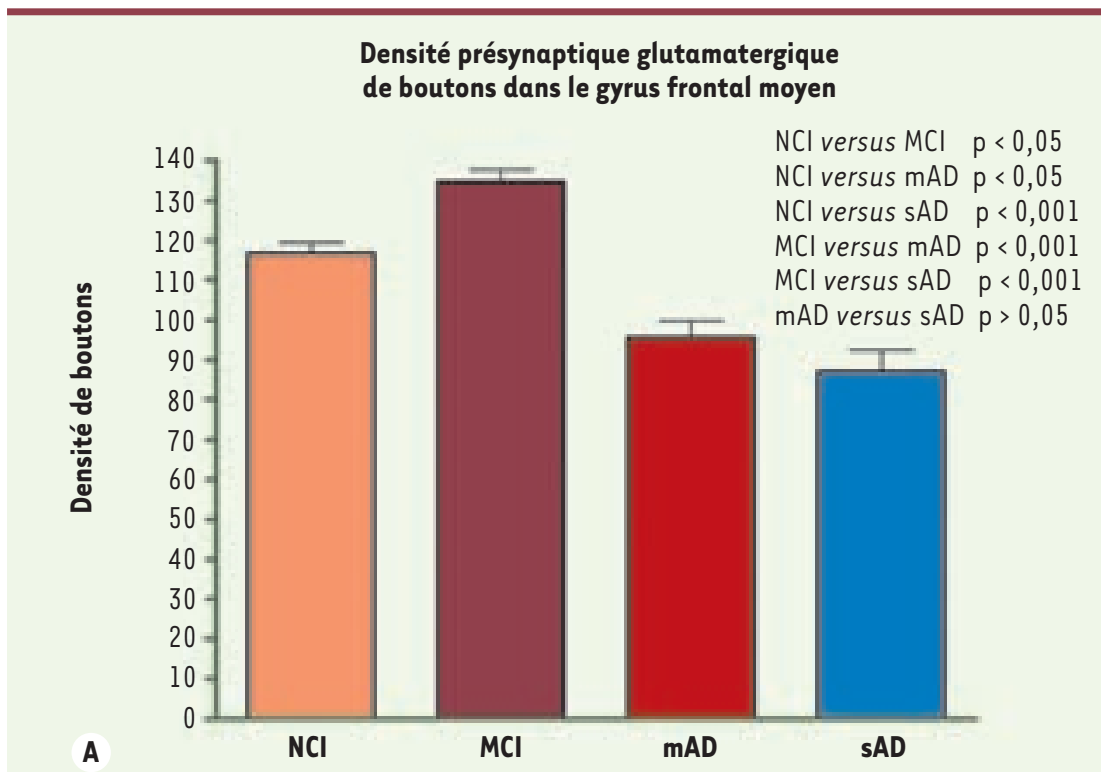

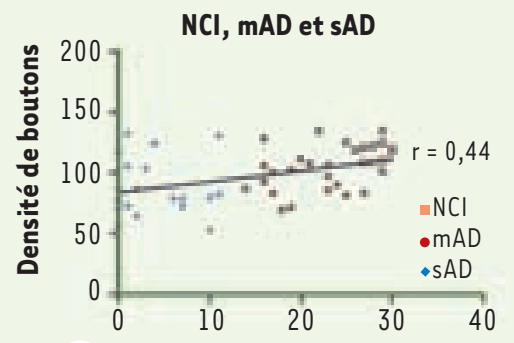

B Mini-examen de l'état mental

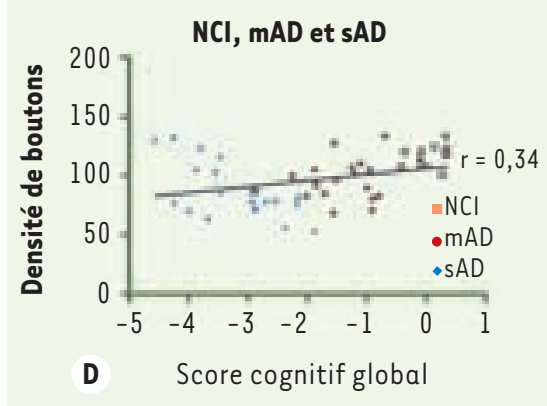

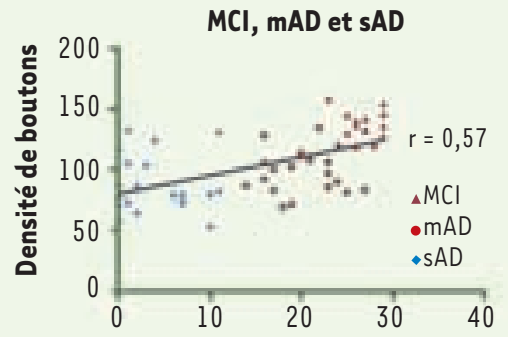

C Mini-examen de l'état mental

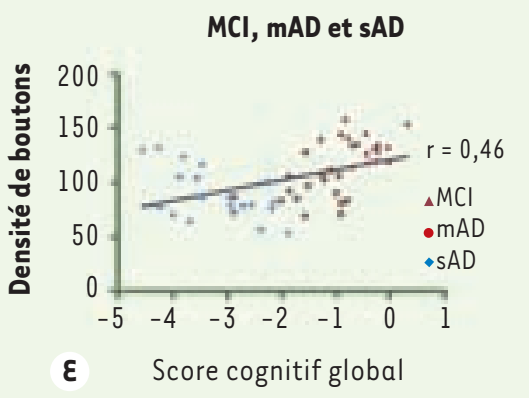

de Mufson, Dekosky et collaborateurs qui ont identifié une augmentation des marqueurs cholinergiques chez les sujets atteints de troubles cognitifs légers [9, 10]. Nous croyons que l'élévation des marqueurs synaptiques pourrait être la manifestation d'une compensation du cerveau destinée à pallier les dommages causés par la présence des molécules $A \beta$ oligomériques.

\section{$\varepsilon$ en conclusion}

Nous avons identifié un remodelage du système des neurotransmetteurs spécifiques au niveau cortical qui montre une étonnante élévation des terminaisons synaptiques glutaminergiques durant la période des troubles cognitifs légers qui précédent la maladie d'Alzheimer. Cette élévation synaptique décline ensuite avec la progression de la maladie jusqu'à atteindre des niveaux inférieurs à la moyenne. Sans doute ces résultats inté-

Figure 1. Description graphique du rapport entre la densité présynaptique glutamatergique de boutons (nombre de boutons présynaptiques par secteur de $1000 \mu \mathrm{m}^{2}$ ) chez des sujets groupés selon leur capacité cognitive. A. Densités présynaptiques glutamatergiques de boutons de gyrus frontal moyen chez des sujets regroupés par capacité cognitive $(\mathrm{NCl}$ : pas de troubles cognitifs; $\mathrm{MCl}$ : troubles cognitifs légers; $\mathrm{mAD}$ : maladie d'Alzheimer associée à des troubles cognitifs modérés; SAD : maladie d'Alzheimer associée à des troubles cognitifs sévères). Encadrés $B$ à $\varepsilon$. Corrélations positives entre la densité de boutons présynaptiques glutamatergiques et I'habileté cognitive testée par le miniexamen de l'état mental $(B, C)$ et avec le score cognitif global $(D, \varepsilon)$. Les données ont été regroupées selon la capacité cognitive: aucun trouble cognitif, états moyens et avancés de la maladie d'Alzheimer $(\mathrm{NCl}, \mathrm{mAD}$ et $S A D: B, D)$ ou des troubles cognitifs légers, et états moyens et avancés de la maladie d'Alzheimer ( $\mathrm{MCl}, \mathrm{mAD}, \mathrm{SAD}: C, \varepsilon)$. À noter : la capacité cognitive décroît avec la diminution de la densité présynaptique glutamatergique à travers tous les regroupements et les types de tests. 


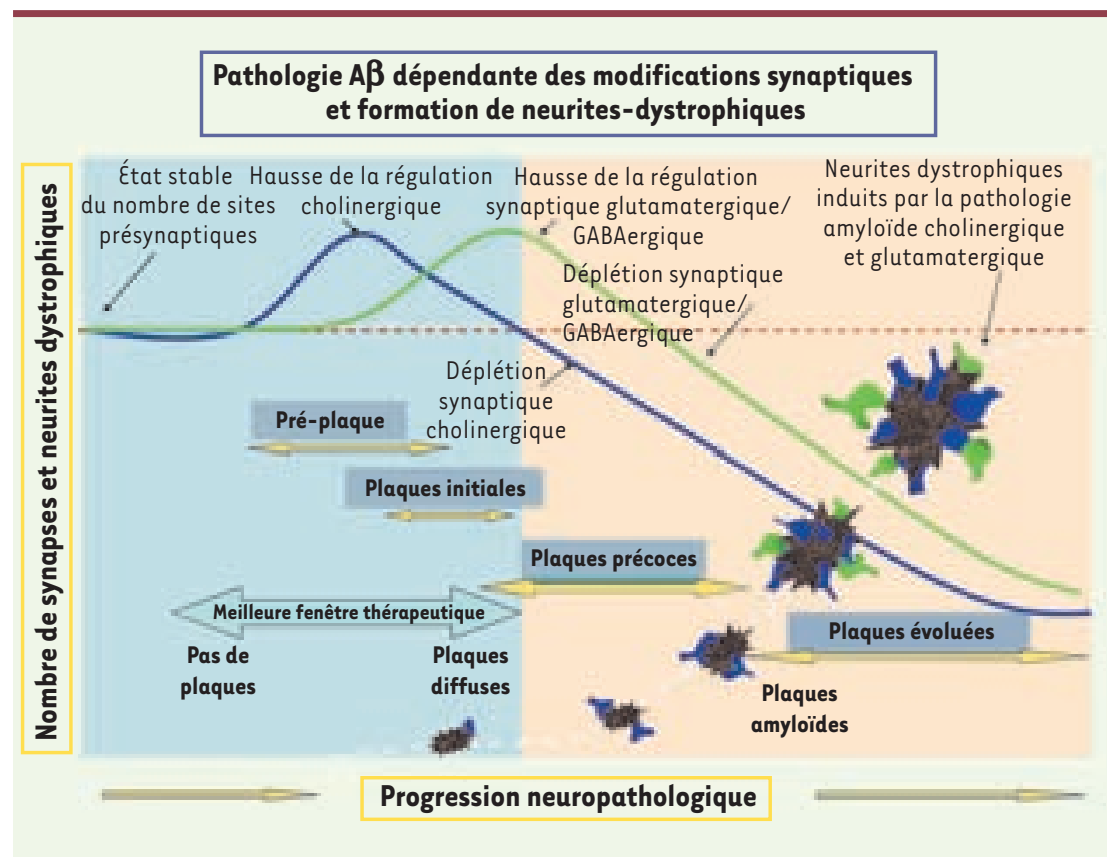

Figure 2. Description de la densité de synaptique et de l'incidence des neurites dystrophiques dans le cortex cérébral au cours de la progression de la pathologie amyloïde ressemblant à la maladie d'Alzheimer dans des modèles animaux transgéniques. On note l'augmentation «paradoxale » de terminaisons synaptiques cholinergiques mais aussi l'augmentation des terminaisons glutamatergiques et GABAergiques dans la première phase de la pathologie amylö̈de, ce à quoi succède une forte déplétion synaptique avec la progression de la pathologie. À droite, on a illustré l'accumulation des neurites dystrophiques en parallèle à la présence des plaques amylö̈des (reproduit de [11] avec la permission de l'éditeur).

resseront les chercheurs mais ils devraient également être utiles aux cliniciens, car, idéalement, la période de compensation pourrait représenter une fenêtre thérapeutique qu'il serait intéressant d'explorer cliniquement avec des médicaments (comme la Mémantine, inhibitrice de la transmission glutamatergique) afin de ralentir la progression de la maladie. $\diamond$

Paradoxical increase

in glutamatergic synaptic terminations in mild cognitive impairment

\section{REMERCIEMENTS}

Nous aimerions remercier le Dr Louis-Étienne Lorenzo pour son aide en traduction, ainsi que Mona-Lisa Bolduc pour son aide administrative.

\section{RÉFÉRENCES}

1. DeKosky ST, Scheff SW. Synapse loss in frontal cortex biopsies in Alzheimer's disease: correlation with cognitive severity. Ann Neurol 1990 ; 27 : 457-64.

2. Terry RD, Masliah $\varepsilon$, Salmon DP, et al. Physical basis of cognitive alterations in Alzheimer's disease: synapse loss is the major correlate of cognitive impairment. Ann Neurol 1991 ; 30 : 572-80.

3. Geddes JW, Monaghan DT, Cotman CW, et al. Plasticity of hippocampal circuitry in Alzheimer's disease. Science $1985 ; 230$ : 1179-81.

4. Geddes JW, Anderson KJ, Cotman CW. Senile plaques as aberrant sprout-stimulating structures. Exp Neurol 1986; $94: 767-76$

5. McKee AC, Kosik KS, Kowall NW. Neuritic pathology and dementia in Alzheimer's disease. Ann Neurol 1991; $30: 156-65$.

6. Bell KF, de Kort GJ, Steggerda S, et al. Structural involvement of the glutamatergic presynaptic boutons in a transgenic mouse model expressing early onset amyloid pathology. Neurosci Lett 2003 ; 353 : 143-7.

7. Bell KF, Ducatenzeiler A, Ribeiro da Silva A, et al. The amyloid pathology progresses in a neurotransmitterspecific manner. Neurobiol Aging 2006; 27 : 1644-57.

8. Bell KF, Bennett DA, Cuello AC. Paradoxical upregulation of glutamatergic presynaptic boutons during mild cognitive impairment. J Neurosci 2007; 27 : 10810-7.

9. DeKosky ST, Ikonomovic MD, Styren SD, et al. Upregulation of choline acetyltransferase activity in hippocampus and frontal cortex of elderly subjects with mild cognitive impairment. Ann Neurol 2002 ; $51: 145-55$.

10. Ikonomovic MD, Mufson $\varepsilon$ J, Wuu J, et al. Cholinergic plasticity in hippocampus of individuals with mild cognitive impairment: correlation with Alzheimer's neuropathology. J Alzheimer Dis 2003 ; 5: 39-48.

11. Bell KFS, Cuello AC Altered synaptic function in Alzheimer's disease. Eur J Pharmacol 2006 ; 545: 11-21.

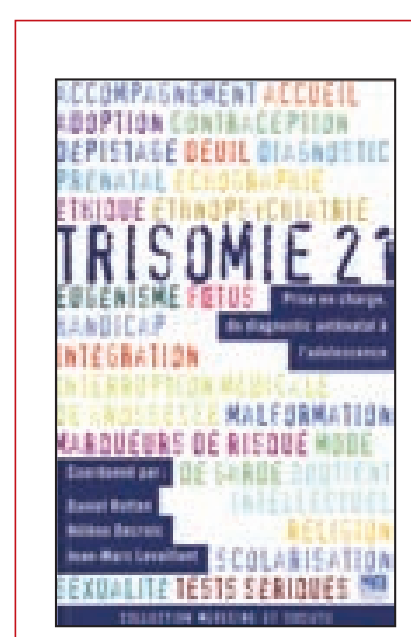

ISBN : 2-84254-105-7 248 pages

\section{Bon de commande}

À retourner à EDK, 2, rue Troyon - 92316 Sèvres Cedex

Tél. : 0155641393 - Fax : 0155641394 - E-mail : edk@edk.fr

NOM :

Prénom :

Adresse :

Code postal :

Ville :

Pays :

Fonction :

Je souhaite recevoir l'ouvrage Trisomie $21: 15 €+3 €$ de port $=\mathbf{1 8} €$ TTC

en ................. exemplaire, soit un total de .......................................... $€$

$\square$ Par chèque, à l'ordre de $\mathbf{E} \mathbf{D} \mathbf{K}$

Par carte bancaire : $\square$ Visa $\square$ Eurocard/Mastercard

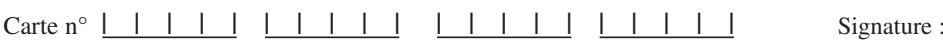

Date d'expiration: $\quad 1 \quad|\quad| 1 \mid$

$\mathrm{N}^{\circ}$ de contrôle au dos de la carte :

.




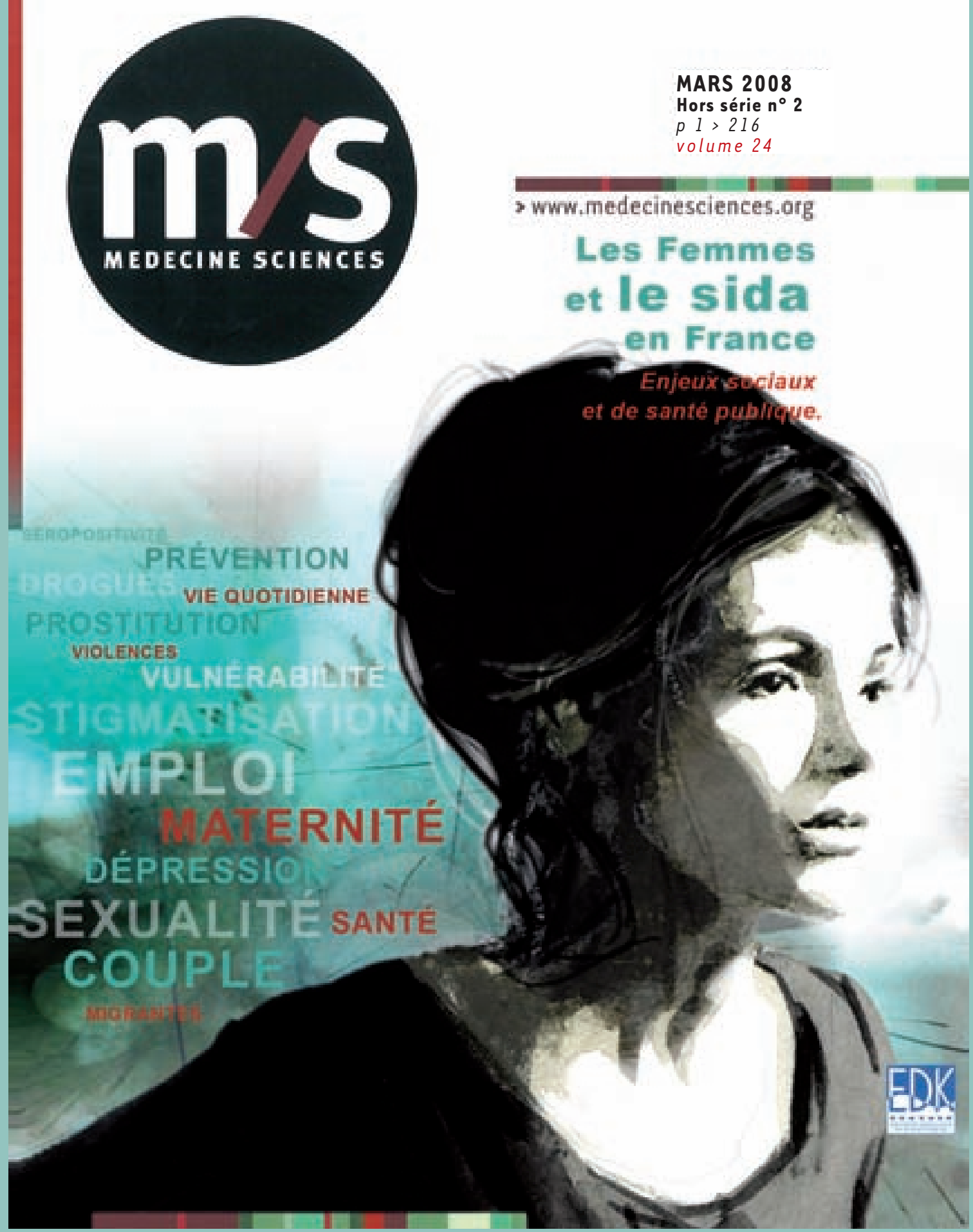

L'Anrs (Agence nationale de recherches sur le sida et les hépatites virales) publie « Les femmes et le sida en France - Enjeux sociaux et de santé publique ». Un numéro indispensable pour comprendre les nouveaux enjeux de prévention dans le contexte de féminisation et de précarisation de l'épidémie et au moment où se pose la question des conditions de vie des femmes atteintes par l'infection du VIH en France.

Un ouvrage précieux, destiné aux chercheurs, élus, décideurs en santé et représentants associatifs.

\section{Bon de commande}

À retourner à EDK, 2, rue Troyon - 92316 Sèvres Cedex

Tél. : 0155641393 - Fax : 0155641394 - E-mail : edk@edk.fr

NOM :

Prénom :

Adresse :

Code postal : .

Ville :

Pays : .

Fonction :

Je souhaite recevoir le hors série n ${ }^{\circ} 2$ de $M / S$ Les femmes et le Sida en France : $18 €+3 €$ de port $=21 €$ TTC en ................. exemplaire, soit un total de

$\square$ Par chèque, à l'ordre de $\mathbf{E} \mathbf{D} \mathbf{K}$

$\square$ Par carte bancaire : $\quad \square$ Visa $\square$ Eurocard/Mastercard

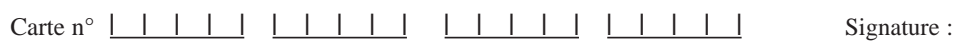

Date d'expiration : $\quad \underline{1 \mid} \mid \underline{1}$

$\mathrm{N}^{\circ}$ de contrôle au dos de la carte : $\quad$ ～～～～ 